\title{
Detection of a balanced translocation carrier through trophectoderm biopsy analysis: a case report
}

\author{
Olga Tšuiko ${ }^{1 *} \mathbb{D}$, Tuuli Dmitrijeva ${ }^{2,3}$, Katrin Kask ${ }^{3}$, Pille Tammur ${ }^{4}$, Neeme Tõnisson ${ }^{5,6}$, Andres Salumets ${ }^{1,7,8,9}$ and \\ Tatjana Jatsenko'
}

\begin{abstract}
Background: Balanced translocation carriers are burdened with fertility issues due to improper chromosome segregation in gametes, resulting in either implantation failure, miscarriage or birth of a child with chromosomal disorders. At the same time, these individuals are typically healthy with no signs of developmental problems, hence they often are unaware of their condition. Yet, because of difficulties in conceiving, balanced translocation carriers often turn to assisted reproduction, some of whom may also undergo preimplantation genetic testing for aneuploidy (PGT-A) to improve the likelihood of achieving a successful pregnancy.

Case report: We describe a female patient, who pursued in vitro fertilization (IVF) treatment coupled with PGT-A following two consecutive miscarriages, unaware of her genetic condition. PGT-A was performed on blastocyststage embryos and the results of comprehensive chromosome screening from a first IVF cycle demonstrated reciprocal segmental aberrations on chromosome 7 and chromosome 10 in two out of four embryos. Due to distinct embryo profiles, the couple was then referred for genetic counselling and subsequent parental karyotyping revealed the presence of a previously undetected balanced translocation in the mother.

Conclusions: These results confirm previous reports that genome-wide PGT-A can facilitate the identification of balanced translocation carriers in IVF patients, providing explanation for poor reproductive outcome and allowing adjustments in treatment strategies.
\end{abstract}

Keywords: Balanced translocations, Preimplantation genetic testing, PGT-A, IVF, Next-generation sequencing

\section{Background}

Any chromosomal aberrations in prospective parents, especially translocations, are often associated with reproductive failure, regardless of parental age. Balanced translocations can be categorized into reciprocal translocations, characterized by the exchange of genetic material between the two non-homologous chromosomes, and Robertsonian translocations, which occur as a result of a fusion of two acrocentric chromosomes (chromosomes 13, 14, 15, 21 or 22). Both reciprocal and Robertsonian translocations are one of the most common chromosomal abnormalities and the estimated frequency of all balanced structural rearrangements in the general

\footnotetext{
* Correspondence: olga.tsuiko@ccht.ee

${ }^{1}$ Competence Centre on Health Technologies, Tiigi 61b, 50410 Tartu, Estonia Full list of author information is available at the end of the article
}

population is $0.2-0.4 \%[1,2]$. Because there is very little or no loss of genetic material, balanced translocation carriers are phenotypically healthy, but they are at increased risk of having fertility issues. The defective chromosome segregation during meiosis can lead to unbalanced karyotype in the germ cells that can be transmitted to the embryo [3-5], resulting in either recurrent miscarriages or birth of a child with severe congenital disorders [6, 7]. Most translocation carriers are unaware of their condition, until parental karyotyping or genetic analysis of either aborted foetus or affected newborn is performed. Once diagnosed, more personalized treatment strategies are offered, and such couples can opt for the use of gamete donation or in vitro fertilization (IVF) treatment coupled with preimplantation genetic diagnosis (now also known as

(c) The Author(s). 2019 Open Access This article is distributed under the terms of the Creative Commons Attribution 4.0 International License (http://creativecommons.org/licenses/by/4.0/), which permits unrestricted use, distribution, and reproduction in any medium, provided you give appropriate credit to the original author(s) and the source, provide a link to the Creative Commons license, and indicate if changes were made. The Creative Commons Public Domain Dedication waiver (http://creativecommons.org/publicdomain/zero/1.0/) applies to the data made available in this article, unless otherwise stated. 
preimplantation genetic testing for structural rearrangements, or PGT-SR) to increase the chance of a successful pregnancy [8-11]. Following PGT-SR, only chromosomally balanced embryos are chosen for transfer based on the results of genetic analysis.

Here, we report a couple, who turned to assisted reproduction after experiencing two consecutive pregnancy losses. The couple chose to undergo preimplantation genetic testing for aneuploidy (PGT-A) to increase the likelihood of pregnancy per embryo transfer and reduce the risk of miscarriage. Comprehensive chromosome screening (CCS) revealed distinct copy-number changes on chromosome 7 and chromosome 10 in trophectoderm biopsies of two IVF embryos, which subsequently led to retrospective identification of a balanced translocation in the mother. In total, the patient underwent two IVF/PGT-A cycles and in the second cycle a pregnancy was established following the transfer of an euploid embryo, resulting in a birth of a healthy baby.

\section{Case presentation \\ Material and methods \\ Patients}

We report a 26-year-old female and her 28-year-old healthy male partner, who experienced difficulties in becoming pregnant since 2015. Female patient had a regular menstrual cycle, but was previously diagnosed with endometriosis in 2012 following laparoscopy, for which she received treatment with goserelin acetate implant (Zoladex ${ }^{\circ}$ ). In January and October 2016, the couple experienced two first trimester miscarriages after natural conception at 5/6 weeks (gestational sac and yolk sac were visible by obstetric ultrasonography) and at $4 / 5$ weeks (only gestational sac was visible) of gestation, respectively. The couple then turned to assisted reproduction in 2017 due to fertility issues. Because of history of endometriosis, the female patient underwent laparoscopy again in April 2017, but no endometriotic lesions were found and fallopian tubes were patent. The female patient was then followed up for multiple cycles for the presence of a dominant follicle. In addition, she was administered with alpha chorionic gonadotropin $\left(\right.$ Ovitrelle $^{\circ}$ ) and dihydrogesterone (Duphaston ${ }^{\circ}$ ) but failed to conceive. In September 2017, the couple enrolled into IVF/PGT-A program at fertility clinic at West-Tallinn Central Hospital for elective embryo transfer to assist in achieving a successful pregnancy. An informed consent was also obtained, allowing to use supernumerary/affected embryos for research purposes.

\section{IVF treatment and embryo biopsy}

Controlled ovarian stimulation was performed using recombinant follicle-stimulating hormone, followed by a gonadotropin-releasing hormone $(\mathrm{GnRH})$ antagonist protocol. Final oocyte maturation was triggered by human chorionic gonadotropin administration $36-38 \mathrm{~h}$ prior to oocyte retrieval. In total 19 oocytes have been retrieved and all of them were fertilized by conventional IVF. The presumed zygotes were then cultured in a SAGE-1 single step media (Origio, Denmark) until day 5 blastocyst stage. Subsequent embryo morphological evaluation was performed according to the criteria set by Gardner and Schoolcraft [12]. Trophectoderm (TE) biopsy was performed on four embryos that reached the blastocyst stage using RI Saturn 5 Active $^{\mathrm{tm}}$ Laser and on average $5-10$ cells were aspirated per embryo. Following TE biopsy, all blastocysts were vitrified using MediCult Vitrification Cooling medias (Origio).

\section{Comprehensive chromosome screening}

For PGT-A, commercially available VeriSeq PGS kit (Illumina Inc., USA) was used for next-generation sequencing (NGS)-based aneuploidy screening. Briefly, TE biopsies were whole-genome amplified (WGA) according to ligation-mediated PCR-based SurePlex protocol (Illumina Inc., USA). The quality of WGA products was controlled on 1.5\% agarose gel and the amount of amplified material was quantified by Qubit dsDNA HS Assay kit (Thermo Fisher Scientific, USA). Next, successfully amplified samples were used for library preparation, according to the manufacturer's VeriSeq PGS kit protocol, and were sequenced on the Illumina MiSeq system. Subsequent CCS was performed using Illumina BlueFuse Multi v4.3 software with an embedded aneuploidy calling algorithm. Based on TE biopsy results, embryo classification was performed according to Preimplantation Genetic Diagnosis International Society (PGDIS) guidelines and recommendations for embryo prioritization (PGDIS, 2016).

\section{Cytogenetic karyotyping}

For blood cell karyotyping, conventional GTG-banding technique (G-bands by trypsin using Giemsa; band level 550) was used for staining metaphase chromosomes from peripheral blood lymphocytes. Chromosome aberrations were classified according to the International System for Human Cytogenetic Nomenclature (ISCN2016).

\section{Results}

The initial PGT-A analysis was performed on four TE biopsies, from which one embryo was predicted to be euploid (Embryo 2); two had recurrent segmental aberrations, involving distal regions of chromosomes 7 and 10 (Embryo 3 and Embryo 4); and one (Embryo 1) had a chaotic profile, characterized by multiple chromosome gains and losses (Table 1). Because of the distinct nature of detected segmental aberrations, which is common to translocation carriers, the couple was referred to genetic 
Table 1 Results of preimplantation genetic testing for aneuploidy (PGT-A)

\begin{tabular}{|c|c|c|c|}
\hline IVF cycle & Embryo ID & Embryo Grade & PGT-A result \\
\hline \multirow[t]{4}{*}{1} & 1 & $5 A B$ & Chaotic with multiple chromosome gains and losses \\
\hline & 2 & $5 A B$ & $46, X X / 46, X X, t(7 ; 10)(q 21.11 ; q 11.23)^{a}$ \\
\hline & 3 & $4 \mathrm{BB}$ & 46,XY,del (7)(pter-q21.11)/dup (10)(pter-q11.23) \\
\hline & 4 & $5 A B$ & 46,XX,dup (7)(q21.11-qter)/del (10)(q11.23-qter) \\
\hline \multirow[t]{3}{*}{2} & 5 & $4 A B$ & 46,XX,del (3)(q26.1-q29) \\
\hline & 6 & $4 A B$ & $\begin{array}{l}45, X Y,-6 \text {, del (7)(pter-q21.11)/dup (10)(pter-q11.23); multiple } \\
\text { mosaic aneuploidies }\end{array}$ \\
\hline & 7 & $5 B B$ & $46, X X / 46, X X, t(7 ; 10)(q 21.11 ; q 11.23)^{a}$ \\
\hline
\end{tabular}

${ }^{\mathrm{a} E u p l o i d ~ e m b r y o s ~ w e r e ~ r e-c l a s s i f i e d ~ a f t e r ~ t h e ~ d e t e c t i o n ~ o f ~ b a l a n c e d ~ t r a n s l o c a t i o n ~ i n ~ t h e ~ m o t h e r ~}$

counselling, and parental karyotyping confirmed the presence of a reciprocal translocation in the mother (Fig. 1). As translocation carriers exhibit alternations in meiosis, segregation of quadrivalents during the meiotic division can produce germ cells with normal and balanced translocated karyotypes, and/or cells with unbalanced karyotype, according to adjacent I or adjacent II modes or 3:1 chromosomal segregation [13]. Such meiotic chromosome segregation patterns have also been previously observed in single sperm cells, derived from male translocation carriers [5], but depending on chromosomes involved, the overall proportion of different meiotic segregation modes can vary between male and female carriers [3]. In our case, the data indicated that Embryo 3 and 4 have inherited one normal and one derivative chromosome from the mother via adjacent II

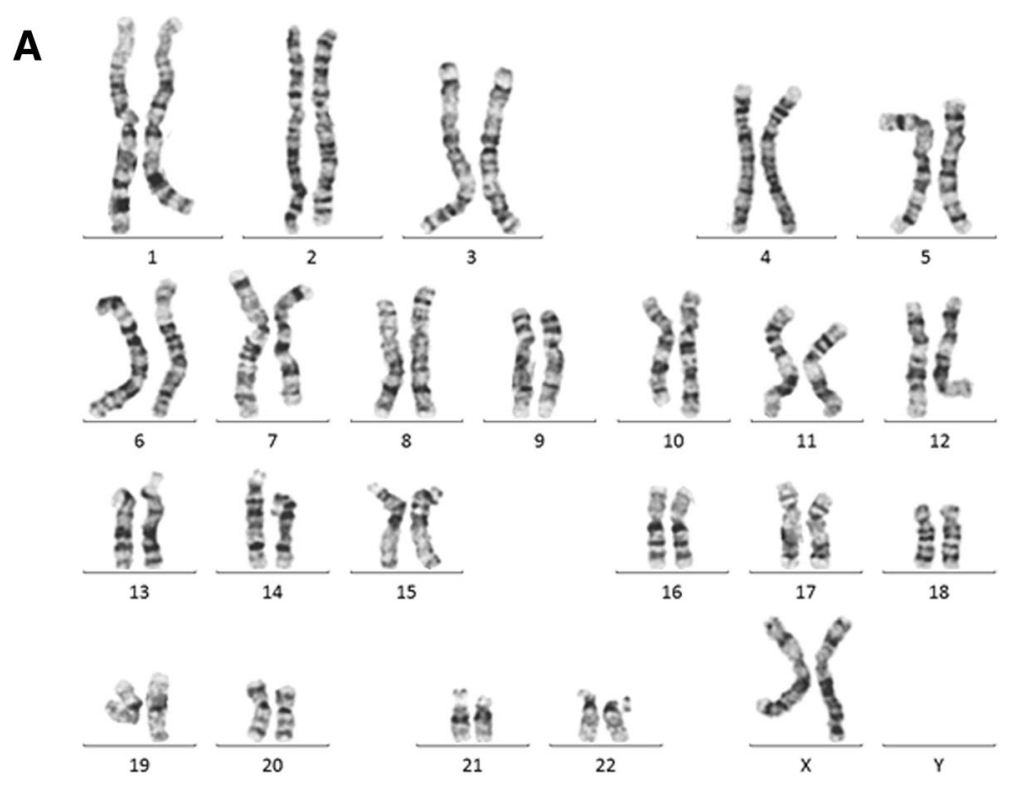

B

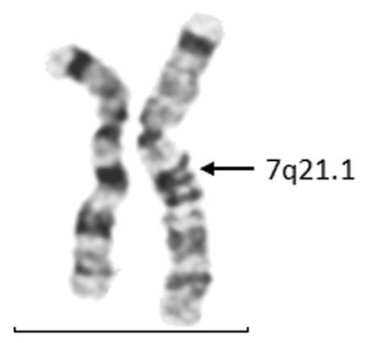

7

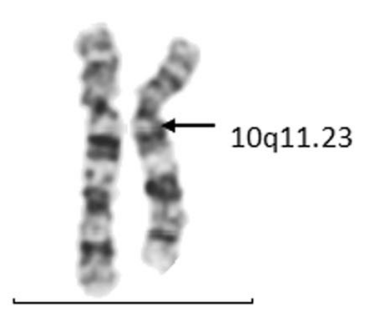

10

Fig. 1 Maternal karyotype. Chromosome banding retrospectively revealed a translocation $46, X X, t(7 ; 10)(q 21.11 ; q 11.23)$ in the mother. a Full maternal karyotype. $\mathbf{b}$ Detailed representation of translocated chromosomes 7 and 10. Arrows indicate breakpoints in rearranged chromosomes 
and adjacent I segregation, respectively (Fig. 2). Due to the diagnosis of a balanced translocation in the mother, the only euploid embryo from the first IVF cycle was reclassified as either $46, \mathrm{XX}$ or $46, \mathrm{XX}, \mathrm{t}(7 ; 10)(\mathrm{q} 21.11 ; \mathrm{q} 11.23)$ and transferred, but without resulting in pregnancy. Because most reciprocal translocations have various segregation modes, depending on chromosomes involved, localization of breakpoints and translocated segment sizes and carrier gender $[14,15]$, empirical data on the risks for viable unbalanced offspring can be lacking, and for each individual couple risk estimation is based on the detected balanced translocation and family history. In the current case, no further counselling was requested by the couple after the diagnosis of a balanced translocation in the mother, and the female patient continued with IVF treatment and embryo testing. In the second cycle, following the same stimulation protocol, three embryos were biopsied and analysed (Table 1). Although Embryo 6 presented with monosomy 6 and multiple aneuploidies, segmental rearrangements of chromosome 7 and 10 were also detected, signifying the defective meiotic segregation patterns of translocated chromosomes. Based on the outcome of embryo chromosome screening from the second IVF cycle, Embryo 7 with a balanced 46,XX/46,XX,

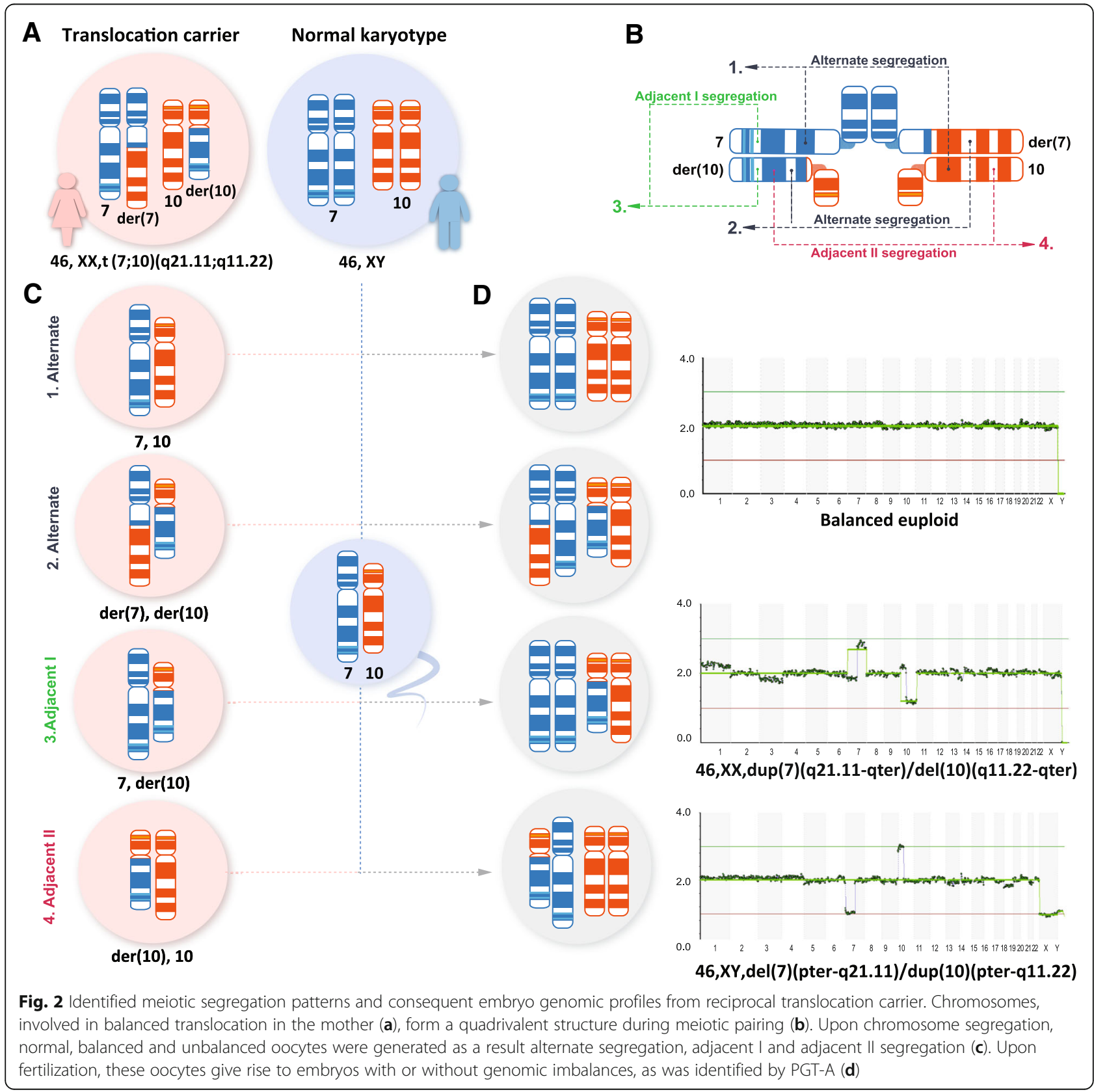


$\mathrm{t}(7 ; 10)(\mathrm{q} 21.11 ; \mathrm{q} 11.23)$ karyotype was chosen for transfer, resulting in a singleton pregnancy and live birth in December 2018.

\section{Discussion}

In this case report, we described a retrospective identification of a balanced reciprocal translocation carrier, based on the embryonic profiles obtained after PGT-A. This discovery together with diagnosed endometriosis provided an extensive aetiology on reproductive failure in the female patient. We have also corroborated previous reports, indicating that PGT-A can identify patients at risk of carrying balanced genomic rearrangements upon observations made in the embryos [16-18].

The frequency of adverse reproductive outcomes can reach up to $5 \%$ in translocation carriers, compared to $<1 \%$ in general population [19]. Even though subfertile couples, undergoing IVF treatment, have an increased prevalence of structural chromosomal rearrangements in comparison to a general population [20-25], routine prenatal karyotyping is not a part of the standard IVF work-up. Instead, selective karyotyping can be indicated for patients with recurrent implantation failure [25] or recurrent pregnancy loss (RPL) [26], which was generally defined as $\geq 3$ consecutive miscarriages [27]. Because our patient had a history of endometriosis, which was considered as a confounding factor, and experienced two early consecutive pregnancy losses, she was not referred for genetic testing based on the genetic counselling guidelines in Estonia that adopted the previously existing RPL definition. However, there has been a significant debate regarding the definition of RPL and patient management, and recent ESHRE guidelines recommend that a diagnosis of RPL should be considered after $\geq 2$ consecutive pregnancy losses [28]. Since miscarriage poses a tremendous psychological burden for any couple, especially to female patients, IVF/ PGT-A option may still seem attractive, despite the controversy and high costs [29]. However, adequately adopted selective karyotyping prior to IVF, especially in patients with a history of miscarriages and/or in cases of severe male infertility, can hold additional clinical and financial benefits [26]. First, parental karyotyping can provide the genetic cause of infertility, as chromosomal abnormalities can lead to gametogenesis failure [30-32]. This information can be important to patients and can aid in patient management and informed decision making for best treatment options. Second, in case of identified structural rearrangements, such as balanced translocations, PGT-SR can be indicated to avoid the transfer of an affected embryo with unbalanced karyotype $[8,10]$. Unlike PGT-A, PGTSR involves diagnosis of inherited structural rearrangements in the embryo, thus it is more likely to be reimbursed by national healthcare systems.
Traditionally, PGT-SR has been performed using fluorescent in situ hybridization, but genome-wide screening via array comparative genomics hybridization or NGS can be performed. Although conceptually PGT-SR is different from PGT-A, comprehensive chromosome screening of the whole genome can have an additional diagnostic value, as it allows to detect other chromosomal imbalances, unrelated to parental translocation, that would normally be missed by targeted approaches. In our case, Embryo 5 had a balanced profile for chromosomes 7 and 10, but a full segmental deletion on chromosome 3 was also detected. Nowadays, NGS techniques with increased sensitivity and resolution are very rapidly implemented into the clinical practice for full genome screening, as they can also detect chromosomal mosaicism [33]. Although extensive knowledge about the effect of mosaic aneuploidies on pregnancy is lacking, this approach allows to perform embryo ranking and subsequent transfer of the most viable embryos first, based on their genomic content and degree of mosaicism [34]. However, chromosomal mosaicism at blastocyst stage still represents a major clinical challenge in patient management, especially when only mosaic embryos are available for transfer.

In conclusion, we reported a case of retrospective balanced translocation carrier identification via blastocyst biopsy analysis. Given that conventional karyotyping is not routinely performed in fertility treatment, the increased use of PGT-A will likely facilitate the detection of undiagnosed balanced translocation carriers among IVF patients.

\section{Abbreviations \\ CCS: Comprehensive chromosome screening; IVF: In vitro fertilization; NGS: Next-generation sequencing; PGT-A: Preimplantation genetic testing for aneuploidy; PGT-SR: Preimplantation genetic testing for structural rearrangements; RPL: Recurrent pregnancy loss; TE: Trophectoderm; WGA: Whole genome amplification \\ Acknowledgments \\ We would like to thank the couple, who participated in this study.}

\section{Authors' contributions \\ OT and TJ performed PGT-A laboratory procedures and wrote the original manuscript. TD performed IVF-related laboratory procedures and embryo bi- opsy. KK was responsible for clinical management of the couple. PT per- formed parental karyotyping and provided interpretation of genetic testing. NT was responsible for genetic counselling. AS supervised the study and co- ordinated the preparation of the manuscript. All authors participated in manuscript revision. All authors read and approved the final manuscript.}

\section{Funding}

This work was supported by the Estonian Ministry of Education and Research (IUT34-16), Enterprise Estonia (EU48695), and the Horizon 2020 innovation program (WIDENLIFE, 692065). NT was supported from PRG555 personal grant.

Availability of data and materials

Data generated and analysed during the study are included in this published case report. Full datasets used in the current study are available from the corresponding author on reasonable request. 


\section{Ethics approval and consent to participate}

This work was approved by the Research Ethics Committee of the University of Tartu (Approval 267/T-2). The coupled signed an informed consent prior to enrolment in the study.

\section{Consent for publication}

The couple gave their written consent for the publication of their clinical case.

\section{Competing interests}

The authors declare that they have no competing interests.

\section{Author details}

${ }^{1}$ Competence Centre on Health Technologies, Tiigi 61b, 50410 Tartu, Estonia. ${ }^{2}$ BioEximi OÜ, Sõle 23, 10614 Tallinn, Estonia. ${ }^{3}$ Women's Clinic, West-Tallinn Central Hospital, Söle 23, 10614 Tallinn, Estonia. ${ }^{4}$ Department of Clinical Genetics, United Laboratories, Tartu University Hospital, L. Puusepa 2, 51014 Tartu, Estonia. ${ }^{5}$ Department of Clinical Genetics in Tallinn, United Laboratories, Tartu University Hospital, L. Puusepa 2, 51014 Tartu, Estonia. ${ }^{6}$ Estonian Genome Center, University of Tartu, Riia 23b, 51010 Tartu, Estonia. ${ }^{7}$ Institute of Bio- and Translational Medicine, University of Tartu, Ravila 19, 50411 Tartu, Estonia. ${ }^{8}$ Department of Obstetrics and Gynaecology, University of Tartu, L. Puusepa 8, 50406 Tartu, Estonia. ${ }^{9}$ Department of Obstetrics and Gynaecology, University of Helsinki and Helsinki University Hospital, Haartmaninkatu 2, 00029 Helsinki, Finland.

\section{Received: 9 February 2019 Accepted: 11 June 2019}

Published online: 18 June 2019

\section{References}

1. Van Dyke DL, Weiss L, Roberson JR, Babu VR. The frequency and mutation rate of balanced autosomal rearrangements in man estimated from prenatal genetic studies for advanced maternal age. Am J Hum Genet. 1983;35(2): $301-8$

2. Jacobs PA, Browne C, Gregson N, Joyce C, White H. Estimates of the frequency of chromosome abnormalities detectable in unselected newborns using moderate levels of banding. J Med Genet. 1992;29(2):103-8.

3. Lledo B, Ortiz JA, Morales R, Ten J, de la Fuente PE, Garcia-Ochoa C, et al. The paternal effect of chromosome translocation carriers observed from meiotic segregation in embryos. Hum Reprod. 2010;25(7):1843-8.

4. Gianaroli L, Magli MC, Ferraretti AP, Munné S, Balicchia B, Escudero T, et al. Possible interchromosomal effect in embryos generated by gametes from translocation carriers. Hum Reprod. 2002;17(12):3201-7.

5. Tran QT, Jatsenko T, Poolamets O, Tšuiko O, Lubenets D, Reimand T, et al. Chromosomal scan of single sperm cells by combining fluorescenceactivated cell sorting and next-generation sequencing. J Assist Reprod Genet. 2018;36(1):91-7.

6. Idowu D, Merrion K, Wemmer N, Mash JG, Pettersen B, Kijacic D, et al. Pregnancy outcomes following 24-chromosome preimplantation genetic diagnosis in couples with balanced reciprocal or Robertsonian translocations. Fertil Steril. 2015;103(4):1037-42.

7. Sugiura-Ogasawara M, Ozaki Y, Sato T, Suzumori N, Suzumori K. Poor prognosis of recurrent aborters with either maternal or paternal reciprocal translocations. Fertil Steril. 2004;81(2):367-73.

8. Verlinsky Y, Tur-Kaspa I, Cieslak J, Bernal A, Morris R, Taranissi M, et al. Preimplantation testing for chromosomal disorders improves reproductive outcome of poor-prognosis patients. Reprod BioMed Online. 2005;11(2):219-25.

9. Munné S, Sandalinas M, Escudero T, Fung J, Gianaroli L, Cohen J. Outcome of preimplantation genetic diagnosis of translocations. Fertil Steril. 2000; 73(6):1209-18.

10. Fischer J, Colls P, Escudero T, Munné S. Preimplantation genetic diagnosis (PGD) improves pregnancy outcome for translocation carriers with a history of recurrent losses. Fertil Steril. 2010;94(1):283-9.

11. Fiorentino F, Spizzichino L, Bono S, Biricik A, Kokkali G, Rienzi L, et al. PGD for reciprocal and Robertsonian translocations using array comparative genomic hybridization. Hum Reprod. 2011;26(7):1925-35

12. Gardner DK, Lane M, Stevens J, Schlenker T, Schoolcraft WB. Blastocyst score affects implantation and pregnancy outcome: towards a single blastocyst transfer. Fertil Steril. 2000;73(6):1155-8.

13. Zhang S, Lei C, Wu J, Sun H, Zhou J, Zhu S, et al. Analysis of segregation patterns of quadrivalent structures and the effect on genome stability during meiosis in reciprocal translocation carriers. Hum Reprod. 2018;33(4):757-67.
14. Ye $Y$, Qian $Y, X u C$, Jin F. Meiotic segregation analysis of embryos from reciprocal translocation carriers in PGD cycles; 2012.

15. Dul E, van Echten-Arends J, Groen H, Kastrop P, Wissen LA, Engelen J, et al. Can characteristics of reciprocal translocations predict the chance of transferable embryos in PGD cycles? J Clin Med. 2014;3(2):348-58.

16. Treff NR, Forman EJ, Katz-Jaffe MG, Schoolcraft WB, Levy B, Scott RT. Incidental identification of balanced translocation carrier patients through comprehensive chromosome screening of IVF-derived blastocysts. J Assist Reprod Genet. 2013;30(6):787-91.

17. Sundheimer LW, Liu L, Buyalos RP, Hubert G, Al-Safi Z, Shamonki M. Diagnosis of parental balanced reciprocal translocations by trophectoderm biopsy and comprehensive chromosomal screening. J Assist Reprod Genet. 2018;35(1):165-9.

18. Robinson K, Neitzel D, Reinwald J, Zhu M, Faulkner N. Identification of previously unknown balanced translocation carriers through routine preimplantation genetic screening using a targeted next-generation DNA sequencing (NGS) assay. Fertil Steril. 2017;107(3):e49.

19. De Braekeleer M, Dao TN. Cytogenetic studies in couples experiencing repeated pregnancy losses, Human Reproduction. 1990;5(5):519-28.

20. Kayed HF, Mansour RT, Aboulghar MA, Serour Gl, Amer AE, Abdrazik A. Screening for chromosomal abnormalities in 2650 infertile couples undergoing ICSI. Reprod BioMed Online. 2006;12(3):359-70.

21. Popovic M, Dheedene A, Christodoulou C, Taelman J, Dhaenens L, Van Nieuwerburgh F, et al. Chromosomal mosaicism in human blastocysts: the ultimate challenge of preimplantation genetic testing? Hum Reprod. 2018; 33(7):1342-54

22. Clementini E, Palka C, lezzi I, Stuppia L, Guanciali-Franchi P, Tiboni GM. Prevalence of chromosomal abnormalities in 2078 infertile couples referred for assisted reproductive techniques. Hum Reprod. 2005;20(2):437-42.

23. Raziel A, Friedler S, Schachter M, Kasterstein E, Strassburger D, Ron-El R. Increased frequency of female partner chromosomal abnormalities in patients with high-order implantation failure after in vitro fertilization. Fertil Steril. 2002;78(3):515-9.

24. Stern C, Pertile M, Norris H, Hale L, Baker HWG. Chromosome translocations in couples with in-vitro fertilization implantation failure. Hum Reprod. 1999; 14(8):2097-101.

25. De Sutter P, Stadhouders R, Dutré M, Gerris J, Dhont M. Prevalence of chromosomal abnormalities and timing of karyotype analysis in patients with recurrent implantation failure (RIF) following assisted reproduction. Facts Views Vis Obgyn. 2012;4(1):59-65.

26. van den Boogaard E, Hermens RPMG, Verhoeve HR, Kremer JAM, van der Veen $F$, Knegt AC, et al. Selective karyotyping in recurrent miscarriage: are recommended guidelines adopted in daily clinical practice? Hum Reprod. 2011;26(8):1965-70.

27. Jauniaux E, Farquharson RG, Christiansen OB, Exalto N. Evidence-based guidelines for the investigation and medical treatment of recurrent miscarriage. Hum Reprod. 2006;21(9):2216-22.

28. Bender Atik R, Christiansen OB, Elson J, Kolte AM, Lewis S, Middeldorp S, et al. ESHRE guideline: recurrent pregnancy loss. Hum Reprod Open. 2018;2018(2): hoy004. https://academic.oup.com/hropen/article/2018/2/hoy004/4963604\#.

29. Rosenwaks Z, Handyside AH, Fiorentino F, Gleicher N, Paulson RJ, Schattman $G L$, et al. The pros and cons of preimplantation genetic testing for aneuploidy: clinical and laboratory perspectives. 2018;

30. Pellestor F, Anahory T, Lefort G, Puechberty J, Liehr T, Hedon B, et al. Complex chromosomal rearrangements: origin and meiotic behavior. Hum Reprod Update. 2011;17(4):476-94.

31. Oliver-Bonet M, Benet J, Sun F, Navarro J, Abad C, Liehr T, et al. Meiotic studies in two human reciprocal translocations and their association with spermatogenic failure. Hum Reprod. 2005;20(3):683-8.

32. Mittwoch U, Mahadevaiah SK. Unpaired chromosomes at meiosis: cause or effect of gametogenic insufficiency? Cytogenet Cell Genet. 1992;59(4):274-9.

33. Munné S, Wells D. Detection of mosaicism at blastocyst stage with the use of high-resolution next-generation sequencing. Fertil Steril. 2017;107(5):1085-91.

34. Grati FR, Gallazzi G, Branca L, Maggi F, Simoni G, Yaron Y. An evidencebased scoring system for prioritizing mosaic aneuploid embryos following preimplantation genetic screening. Reprod BioMed Online. 2018;36(4):442-9.

\section{Publisher's Note}

Springer Nature remains neutral with regard to jurisdictional claims in published maps and institutional affiliations. 